\title{
FE-DEM Analysis of the Effect of Tread Pattern on the Tractive Performance of Tires Operating on Sand*
}

\author{
Hiroshi NAKASHIMA**, Yuzuru TAKATSU***, Hisanori SHINONE***, \\ Hisao MATSUKAWA ${ }^{\dagger}$ and Takahiro KASETANI ${ }^{\dagger}$ \\ ** Graduate School of Agriculture, Kyoto University \\ Oiwake-cho, Kitashirakawa, Sakyo-ku, Kyoto 606-8502, Japan \\ E-mail: hiron@kais.kyoto-u.ac.jp \\ *** Graduate School of Agriculture, Kyoto University \\ Oiwake-cho, Kitashirakawa, Sakyo-ku, Kyoto 606-8502, Japan \\ Department of Agricultural \& Environmental Engineering, Faculty of Agriculture, Kyoto University \\ Oiwake-cho, Kitashirakawa, Sakyo-ku, Kyoto 606-8502, Japan
}

\begin{abstract}
Soil-tire system interaction is a fundamental and important research topic in terramechanics. We applied a 2D finite element, discrete element method (FE-DEM), using FEM for the tire and the bottom soil layer and DEM for the surface soil layer. Satisfactory performance analysis was achieved. In this study, to clarify the capabilities and limitations of the method for soil-tire interaction analysis, the tractive performance of real automobile tires with two different tread patterns - smooth and grooved - was analyzed by FE-DEM, and the numerical results compared with the experimental results obtained using an indoor traction measurement system. The analysis of tractive performance could be performed with sufficient accuracy by the proposed 2D dynamic FEDEM. FE-DEM obtained larger drawbar pull for a tire with a grooved tread pattern, which was verified by the experimental results. Moreover, the result for the grooved tire showed almost the same gross tractive effort and similar running resistance as in experiments. However, for a tire with smooth tread pattern, the analyzed gross tractive effort and running resistance behaved differently than the experimental results, largely due to the difference in tire sinkage in FE-DEM.
\end{abstract}

Key words : Soil-Tire System, Interaction, FEM, DEM, Traction, Sand

\section{Introduction}

Soil-tire system interaction is a fundamental research topic in terramechanics. Recent computing developments have enabled us to apply a new approach called computational mechanics, to these interaction problems. Application of the finite element method (FEM) to soil-tire systems assumes that the tire surface is smooth and without the influence of a traction-type tread pattern, because of the complex contact stress boundary at the soil-tire interface $^{(1)-(7)}$. Recently, Oida et al. ${ }^{(8)}$ developed coupling methods for a unified tire-terrain system simulation tool using FEM and the finite volume method (FVM) for their tire design processes, although it does not consider the friction between the tire and terrain. The discrete, or distinct, element method (DEM) ${ }^{(9)}$ has been applied, not only to soil or rock mechanics problems, but also to interaction problems with complex boundary conditions in terramechanics $^{(10)-(13)}$.

Since there is an inherent problem that large computational time is required for contact check and contact reaction calculation in DEM, it is quite natural to utilize both merits of FEM and DEM to compensate for the demerits of each method ${ }^{(14)-(16)}$. For soil-tire interaction analysis, a reduction in DEM calculation time can be expected by reducing the total number 


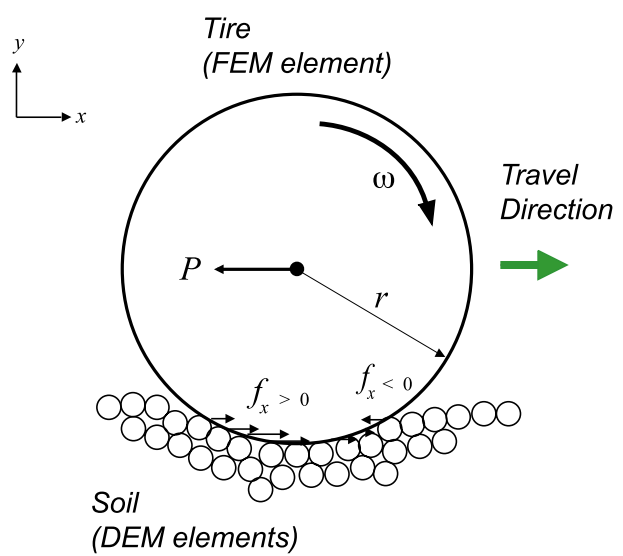

Fig. 1 Horizontal contact reaction at soil-tire interface.

of DEs and replacing them with FEs as much as possible. The treatment of localized shear, which is caused by the action of tire lugs, for example, is realized by DEM, but cannot be directly expressed by conventional FEM. An algorithm for the implementation of dynamic FE-DEM for soil-tire system interactions, with examples of the simple vertical sinkage and horizontal travel of a tire was previously proposed by the authors ${ }^{(16),(17)}$.

The purpose of this study is to apply 2D FE-DEM to the tractive performance analysis of real automobile tires with two different tread patterns - smooth and grooved - and to check the validity of present soil-tire interaction analysis by comparing numerical results with experimental results. Moreover, the effect of tread patterns is discussed, based on the numerical results.

\section{Outline of the Finite Element-Discrete Element Method}

The tractive performance analysis of a tire by FE-DEM is summarized in this section. A detailed explanation of FE-DEM can be found in the previous publication ${ }^{(17)}$.

\subsection{Formulation for FE-DEM}

The tire and subsurface soil are modeled as elastic bodies in FEM. DEM is applied to modeling the upper soil layer, where shear and large deformation of soil under the action of tire or tire treads would be expected.

Subsequently, we must solve two types of equations of motion, one for translation (for both FEM and DEM), and the other for rotation (for DEM), which are represented in vector notation:

$$
\begin{array}{lll}
\boldsymbol{F}_{c}+\boldsymbol{F}_{b} & =m \frac{d^{2} \boldsymbol{u}}{d x^{2}} \quad(\text { for FEM, DEM) } \\
\boldsymbol{N}_{c} & =I \boldsymbol{\Omega} \quad(\text { for DEM })
\end{array}
$$

where $\boldsymbol{F}_{c}$ is the contact reaction, $\boldsymbol{F}_{b}$ is the volumetric force, $m$ is the mass, $\boldsymbol{u}$ is the displacement, $\boldsymbol{N}_{c}$ is the moment by $\boldsymbol{F}_{c}, I$ is the moment of inertia, and $\boldsymbol{\Omega}$ is the angular acceleration. Naturally, there are $x$ - and $y$-components in Eq. (1) and one component in Eq. (2) in the case of a two-dimensional (2D) FE-DEM problem. Explicit time integration is applied to solve the above equations.

\subsection{Traction Performance}

Figure 1 shows the horizontal contact reaction for gross tractive effort and running resistance of a tire in FE-DEM. A tire is assumed to travel to the right with some positive tire slip. Consequently, we can calculate the gross tractive effort $H$ of a tire as follows:

$$
H=\sum f_{x}^{+}
$$




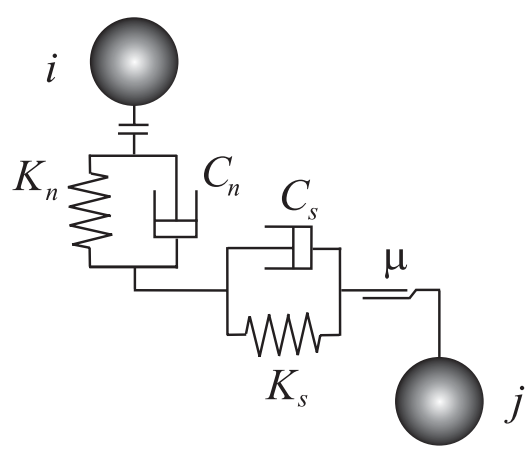

Fig. 2 Contact model for DEM.

and the running resistance $R(<0)$ can be obtained by:

$$
R=\sum f_{x}^{-}
$$

where $f_{x}^{+}$and $f_{x}^{-}$are the positive and negative components of contact reaction at the tire-soil interface acting on the contact nodes of tire FEs with respect to the global coordinate system (Fig.1).

Based on Eqs. (3) and (4), a well-known relationship for drawbar pull $P$ can be calculated as

$$
P=H-|R|
$$

The slip $i$ of a tire can be expressed by the following equation:

$$
i=\frac{V_{w}-V}{V_{w}}=\left(1-\frac{V}{r \omega}\right)
$$

where $V$ is the translation speed of the tire center, $r$ is the rolling radius of a free-rolling tire, and $\omega$ is its angular velocity. The constant angular velocity $\omega$ and constant translational velocity $V$ of a tire are the inputs in our FE-DEM, and the tractive performance is analyzed based on the constant slip value of $i$ defined by Eq. (6).

\subsection{Contact Reaction}

Figure 2 shows a typical contact model in DEM, which consists of a spring and a damper in parallel for contacts in the normal and tangential directions. Each component of reaction in the $j$ direction, i.e., $j=n$ for normal direction or $j=s$ for tangential direction, can be expressed as:

$$
F_{j}=K_{j} \Delta u_{j}+C_{j} \Delta \dot{u}_{j} \quad(j=n, s)
$$

where $F_{j}$ is the contact reaction, $\Delta u_{j}$ is the relative displacement of contacting elements, $K_{j}$ is the spring constant, $C_{j}$ is the damping coefficient, and $\Delta \dot{u}_{j}$ is the relative velocity of the contacting elements. For tangential reaction, Coulomb friction may also be considered, such that $F_{s}=\mu F_{n}$ if $F_{s}>\mu F_{n}$ and $F_{s}=F_{s}^{*}$ if $F_{s}<\mu F_{n}$ with a friction coefficient of $\mu$ and $F_{s}^{*}$ is an updated tangential reaction.

Figure 3 is a contact model at the FE-DEM interface, where we focus on line segment 1-2 of a FE for the 2D case to distribute the contact reaction linearly into nodal reactions $\boldsymbol{F}_{1}$ and $\boldsymbol{F}_{2}$ using contact force $\boldsymbol{R}_{3}$ in DEM. As in the figure, contact between the FE mesh and a DE can be treated, for the most part, for a 2D case, as the contact between DEs with slight modification of the redistribution of contact reaction, by using a shape function on the supporting nodes of a contacting line segment of FE mesh. 


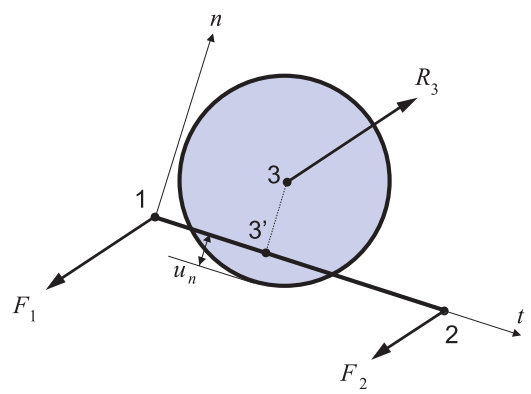

Fig. 3 Contact model of the interface in FE-DEM.

Table 1 Tire data for FE-DEM.

\begin{tabular}{lc}
\hline Tire Size & $165 / 65 \mathrm{R} 13$ \\
Diameter [mm] & 535 \\
Maximum Width [mm] & 175 \\
Tread Width [mm] & 127 \\
Inflation Pressure [kPa] & 140 \\
Vertical Load [N] & 980 \\
\hline
\end{tabular}

\subsection{Program Flow}

The simulation strategy is composed of following three modes: (i) soil consolidation by the weight of DEs at the surface soil layer; (ii) free sinkage of tire FEs onto the soil surface until the vertical contact reaction of the tire reaches the vertical load of the tire; and (iii) tire travel mode with prescribed angular and translational velocities.

\section{Numerical Experiment}

\subsection{Target Tires}

Table 1 shows data for a tire whose geometrical and loading data correspond with the experimental conditions ${ }^{(18)}$. Two typical tread patterns-one smooth (Fig. 4(a)) and one laterally grooved (Fig. 4(b)) - were prepared to compare the experimental results.

The tire deformation is assumed to be negligible for the given experimental inflation pressure, so that the free rolling radius of the tire $r$ can be set to $r=535 \times 0.5=267.5 \mathrm{~mm}$. As in the experiments, the type of soil in the analysis is assumed to be air-dried sand, where no cohesion of soil is assumed.

\subsection{Preparation of Analysis for FE-DEM}

The smooth-treaded tire can be modeled by relatively large FE meshes. However, to precisely express the grooves for the grooved tires, small FE meshes should be prepared around the grooves so that the discretization errors would not affect the existence of grooves in the FE-DEM. Moreover, relatively small DEs were prepared for the surface soil layer, the tire was to travel on. Table 2 summarizes the elemental data of the FE-DEM with respect to the tread condition. The randomly distributed diameter of a circular DE is used in the present FE-

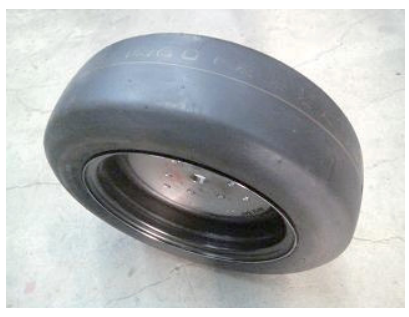

(a)

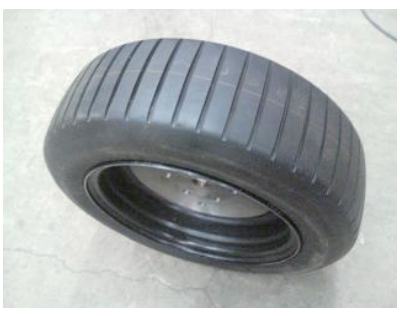

(b)

Fig. 4 Automobile tire with two tread patterns in experiments ${ }^{(18)}$. 
Table 2 Tread pattern and DEM soil data.

\begin{tabular}{lcc}
\hline Tread pattern & Smooth & Grooved \\
Number of tread & - & 56 \\
Depth of tread groove [mm] & - & 6.5 \\
Diameter of DE [mm] (random) & $4,5,6$ & $4,5,6$ \\
Total numbers of DE & 9643 & 9643 \\
Total numbers of FE for tire & 140 & 448 \\
Total numbers of FE node for tire & 155 & 561 \\
Total numbers of FE for bottom soil & 100 & 100 \\
Total numbers of FE node for bottom soil & 134 & 134 \\
\hline
\end{tabular}

Table 3 Material parameters for FE-DEM.

\begin{tabular}{cccccc}
\hline & \multicolumn{2}{c}{ Tire } & Lower Soil & Surface Soil & Wall at SS \\
& Rubber (TR) & Wheel Rim & (LS) & (SS) & (WL) \\
\hline Young's Modulus [MPa] & 1.5 & 206000 & 9.0 & - & - \\
Poisson's Ratio & 0.49 & 0.3 & 0.3 & - & - \\
Elemental Density [kg/m $\left.3 \mathrm{~m}^{3}\right]$ & 1800 & 3000 & 2300 & 2600 & 2600 \\
\hline
\end{tabular}

NB) TR: tire rubber; LS: lower soil; SS: surface soil; WL: wall

DEM, as listed in Table 2. Since the thickness of the DEM layer did not have a critical effect on the tractive performance ${ }^{(17)}$, rather thin layers of DEs were initially arranged to reduce the computation time. Thus, the total number of DEs was 9643 for both tread patterns.

\subsection{Parameters for FE-DEM}

Numerical parameters for FE-DEM are summarized in Tables 3 and 4. Young's modulus for the lower soil was assumed to be $9 \mathrm{MPa}$, as in Table 3. Moreover, the coefficient of internal friction between sand particles is set at 0.6 , while the other coefficient of friction is 0.3 . The damping coefficients for contact reactions were calculated from the critical damping formula.

All the parameters were decided by a trial-and-error preliminary computation in terms of numerical stability. The time step in current explicit time integration was $1.0 \times 10^{-4} \mathrm{~s}$.

\section{Results and Discussion}

\subsection{Tire with Smooth Tread Pattern}

Figure 5 shows an early step of the simulation just after the beginning of travel to the right for a tire with smooth tread pattern, for a slip of $20 \%$. The preparation of DE with randomly generated radius required the flattening of the soil surface by neglecting DEs higher than a prescribed height of soil, to facilitate the definition of the horizontal soil surface after the initial consolidation of soil DEs.

Figure 6 shows a final configuration of the simulation for the tire with a smooth tread pattern. The travel distance is about $1 \mathrm{~m}$. Total elapsed time of the analysis depends on the slip condition. When the tire begins to run, the surface soil deformed and compressed just below the tire, and soil rebound occurred after detaching the tire from the soil. A tire with smooth tread pattern resulted in a relatively flat soil surface after traveling over it, as shown in Fig. 6.

The gross tractive effort and running resistance of a tire with a smooth tread pattern are shown with the corresponding experimental results ${ }^{(18)}$ in Fig. 7. In the figure, filled-circle markers with lines indicate the results of FE-DE simulation and cross markers without lines represent experimental results. The drawbar pull $P$ of tire for FE-DEM can be calculated by

Table 4 Contact parameters for FE-DEM.

\begin{tabular}{ccccc}
\hline & TR-SS & LS-SS & SS & SS-WL \\
\hline$K_{n}[\mathrm{~N} / \mathrm{m}]$ & 150000 & 150000 & 150000 & 150000 \\
$K_{s}[\mathrm{~N} / \mathrm{m}]$ & 75000 & 75000 & 75000 & 75000 \\
$\mu$ & 0.3 & 0.3 & 0.6 & 0.3 \\
\hline
\end{tabular}


and Logistics

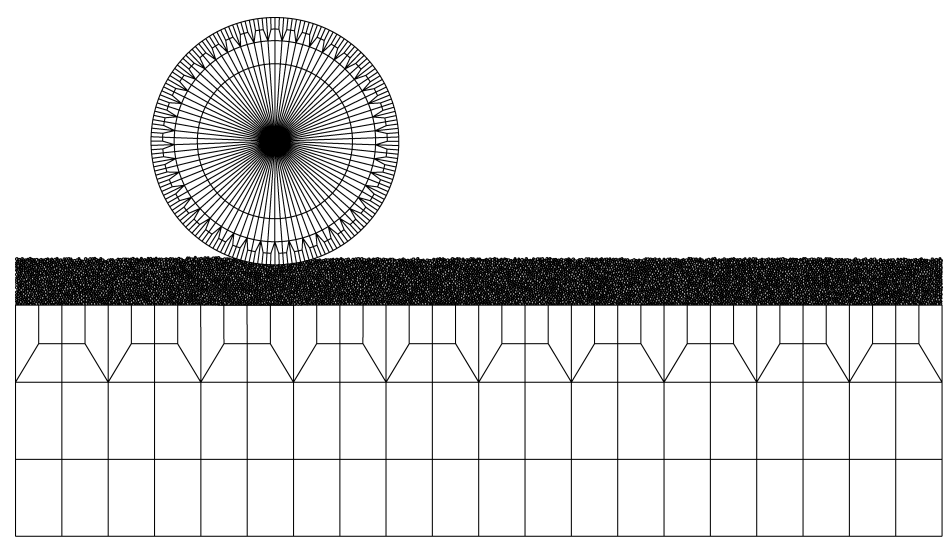

Fig. 5 Configuration for a tire with a smooth tread pattern at the beginning of tire travel.

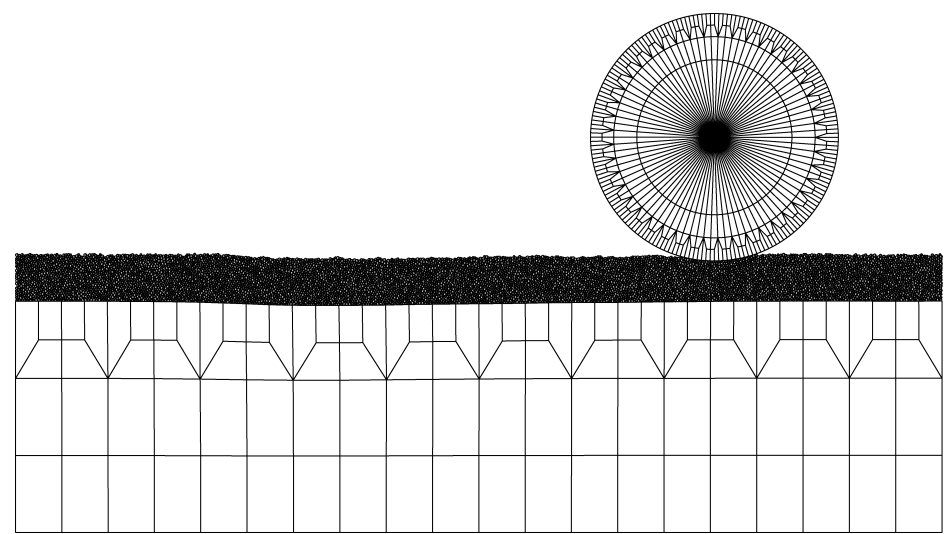

Fig. 6 Final configuration for a tire with a smooth tread pattern.

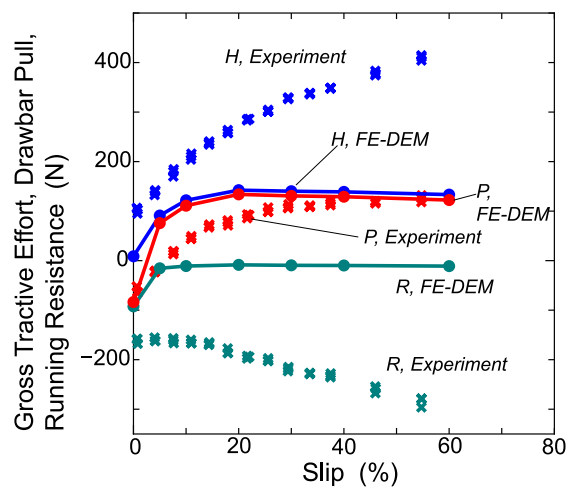

Fig. 7 Tractive performance of a tire with a smooth tread pattern by FE-DEM. 


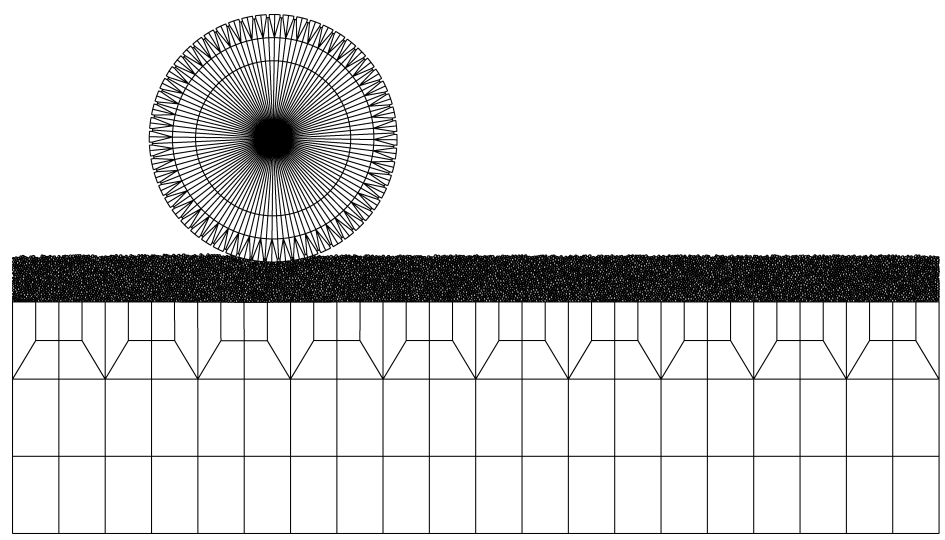

Fig. 8 Configuration for a tire with a grooved tread pattern at beginning of tire travel.

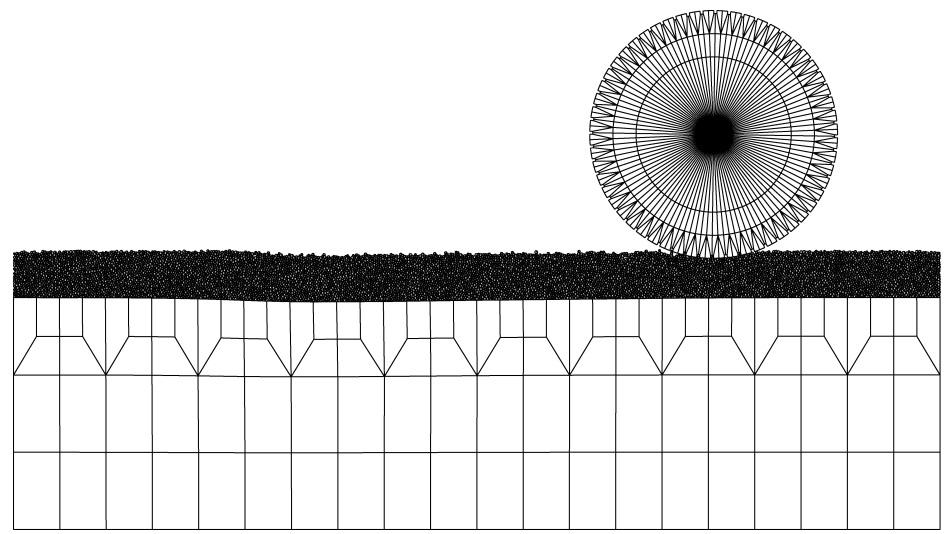

Fig. 9 Final configuration of a tire with a grooved tread pattern.

Eq. (5), as shown in the figure. For small slip, below 10\%, the drawbar pull curve rises rapidly as the slip increases. However, it remains almost constant for a slip range of more than $10 \%$. The behavior of drawbar pull is similar to well-known experimental results ${ }^{(19)}$.

Note that a large difference exists between numerical and experimental results in gross tractive effort and running resistance. However, for the drawbar pull, the difference was canceled out for larger slip, and the results for drawbar pull are not significantly different from the experimental results.

\subsection{Tire with Grooved Tread Pattern}

Figure 8 shows a configuration just after beginning tire travel to the right for $20 \%$ slip, and Fig. 9 depicts the configuration of a tire with grooved tread pattern at the end of the simulation. The travel distance is again about $1 \mathrm{~m}$.

The surface of DE at the left of the initial tire contact swells due to the acceleration of tire travel in Fig. 9. For other terrain surfaces of tire locus, the soil was deformed and compressed near the dead bottom center of tire rotation, and soil rebound occurred after detachment of the tire FE. This is confirmed that some soil DEs are trapped in the grooves of tire FE during tire travel, as shown in Fig. 10. The soil surface after the tire passes shows the remains of the groove shape as seen in Figs 9 and 10.

The gross tractive effort and running resistance of a tire with a grooved tread pattern are shown in Fig. 11. In the figure, filled-circle markers with lines show the results of FE-DE simulation and cross markers without lines represent experimental results. Throughout the slip conditions of the analysis, the gross tractive effort increased as the slip became large. The absolute value of the running resistance decreased for slip up to $10 \%$, and increased in a slip range larger than $10 \%$. Moreover, for slip less than $40 \%$, the drawbar pull became large, and 


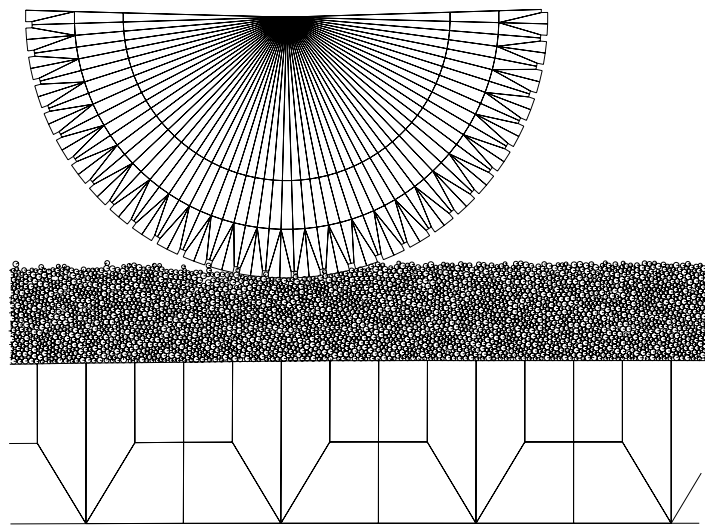

Fig. 10 Close up view of a grooved tire at contact.

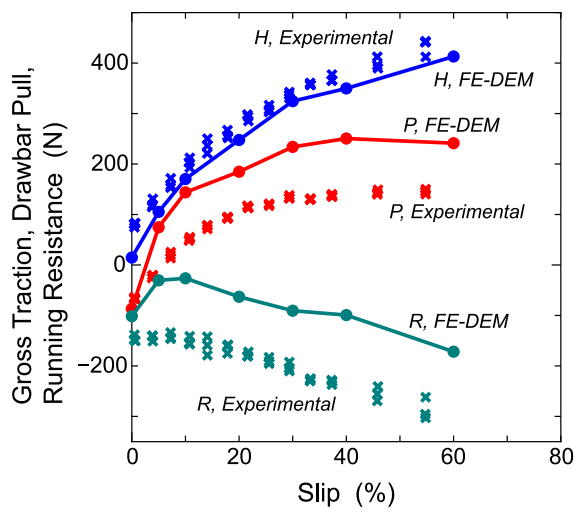

Fig. 11 Tractive performance of a tire with grooved tread pattern by FE-DEM and by experiment.

it remained constant for slip of more than 40\%, as shown in Fig. 11.

Comparing with the results of numerical analysis and experiments, the gross tractive effort in experiments was found to be slightly larger than that in FE-DEM analysis. Moreover, experimental absolute value of running resistance was larger than the numerical result for all the given slip ranges. As a result, the drawbar pull in FE-DEM shows larger value than experimental result. Therefore, the proposed FE-DEM seems to have sufficient accuracy to predict gross tractive effort of a grooved tire on sand.

\subsection{Discussion of the Effect of Tread Pattern}

The experimental result showed that the drawbar pull for the smoothed tire was $125.3 \mathrm{~N}$, while that for the grooved tire was $150.0 \mathrm{~N}$ at $55 \% \mathrm{slip}^{(18)}$. Thus, the grooved tire has superior drawbar pull than the smoothed tire. Moreover, the experimental gross traction was 409.9 $\mathrm{N}$ for the smooth tire and $427.5 \mathrm{~N}$ for the grooved tire at $55 \% \operatorname{slip}^{(18)}$. Similarly, the result from FE-DEM (in Figs 7 and 11) also indicates larger gross traction and drawbar pull for the grooved tire than for the smooth tire.

Numerical drawbar pull for the grooved tire showed a rather slow increase with increasing tire slip, while that for the smooth tire exhibited a rapid increase at low tire slip and remained almost constant for a larger tire slip range. For larger slip conditions, the result of FE-DEM depicts the similar qualitative behavior of drawbar pull as in the experimental result.

However, numerical running resistance was rather flat for increasing tire slip with a smooth tread pattern (Fig. 7), and decreased slightly at small slip, followed by a constant increase in absolute value with respect to slip as in the experimental results for a grooved pattern (Fig. 11).

The difference in behavior of running resistance of a smooth tire result may be attributed 


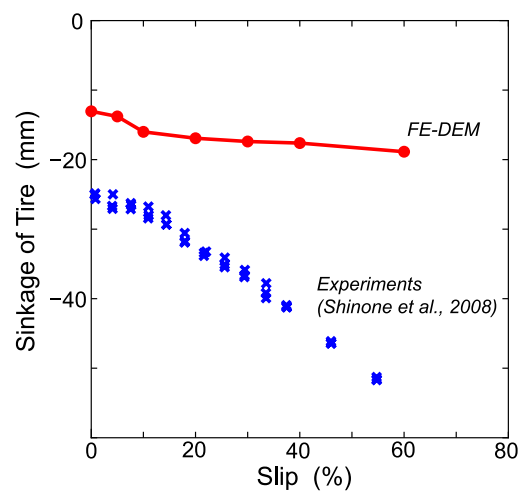

Fig. 12 Sinkage versus slip for a smooth tire.

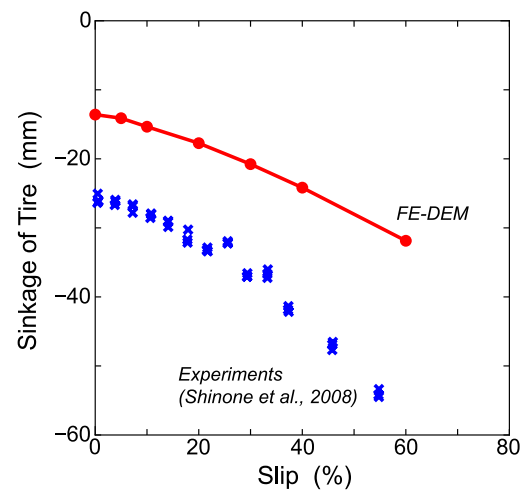

Fig. 13 Sinkage versus slip for a grooved tire.

to a difference in the tire sinkage. Figure 12 shows the relationship of sinkage and tire slip for a smooth tire obtained by FE-DEM. In the figure, the sinkage becomes almost flat with increasing slip.

On the other hand, the sinkage of a tire with grooved tread pattern is shown in Fig. 13. The increasing trend of the absolute value of sinkage is similar to the experimental results. This might be caused by the DEs trapped in grooves, as a result of a combination of the following factors: (i) The elemental friction between DEs, which was larger than the friction between the tire and soil in terms of the coefficient of friction; and (ii) the existence of grooves would cause the removal of some surface DEs through scratching action with the grooves (Fig. 10).

To clarify the role of the groove in the analysis, additional computations for a tire with grooved tread pattern were performed, where the total number of grooves was 28 and total number of nodes and FEs for tire and rim were reduced to 224 and 281, respectively, but the other conditions were the same as in Tables 2-4.

Figure 14 shows the result of tractive performance for different numbers of grooves. It is interesting to note that the tractive performance shows no difference for slip conditions of up to $10 \%$, but the behavior of a 28 -groove tire degraded for slip greater than $10 \%$. For higher slip conditions, the action of the tire surface might be mainly shear over the soil, and this action shows a dependency on the total number of grooves.

The sinkage variation is shown in Fig. 15. Again, it is seen that the sinkage is the same in two cases up to $10 \%$ slip, but the 28 -groove tire shows a weakened effect on sinkage with respect to increasing tire slip after $10 \%$ of slip.

Note that the result of sinkage from experiments is that it increases in absolute value with respect to tire slip for both smooth and grooved tread, and little difference in tread pattern is observed. In the present analysis, a random DE radius was introduced, and this should be more 


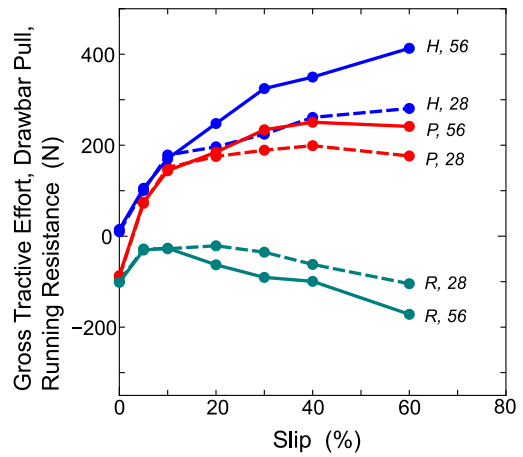

Fig. 14 Traction performance against the total number of grooves.

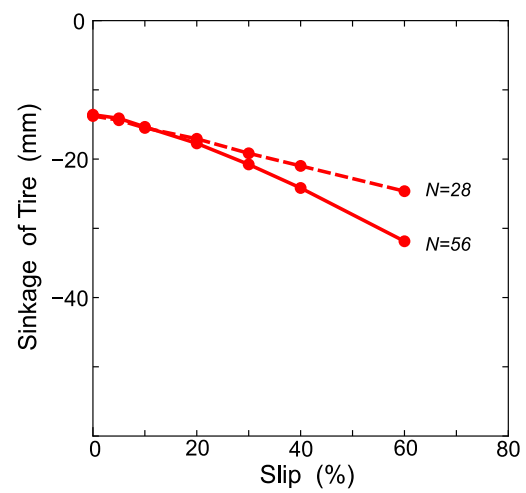

Fig. 15 Sinkage versus the total number of grooves.

effective in increasing tire sinkage at the upper soil layer than in the previous reports ${ }^{(16),(17)}$. However, there is still a limitation of degrees-of-freedom in the 2D analysis of tire-soil contact problems, where the lateral flow of soil could not be considered. Taking this limitation into account, the present 2D FE-DEM had sufficient applicability for determining the effect of smooth and grooved tread patterns on automobile tires.

\section{Conclusion}

The analysis of tractive performance of real automobile tires with both smooth and grooved tread patterns, traveling on sand was performed with sufficient accuracy by the proposed 2D dynamic FE-DEM. Analysis obtained larger drawbar pull for a tire with a grooved tread pattern, which was verified by the experimental results. Moreover, the grooved tire showed almost the same gross tractive effort and similar running resistance as in experiments. However, for a tire with a smooth tread pattern, the analyzed gross tractive effort and running resistance behaved differently than the experimental results, largely due to the difference in tire sinkage in FE-DEM.

\section{References}

( 1 ) Perumpral, J. V., Liljedahl, J. B., Perloff, W. H., A numerical method for predicting the stress distribution and soil deformation under a tractor wheel, Journal of Terramechanics, 8(1)(1971), pp. 9-22.

( 2 ) Aubel, T., FEM-Simulation der Wechselwirkung zwischen Reifen und nachgiebiger Fahrbahn, Automobiltechnische Zeitschrift, 94(1992), pp. 662-667.

( 3 ) Hiroma, T., Wanjii, S., Kataoka, T., Ota, Y., Stress analysis using FEM on stress distribution under a wheel considering friction with adhesion between a wheel and soil, Journal of Terramechanics, 34(4)(1997), pp. 225-233.

( 4 ) Yong, R. N., Fattah, E. A., Boonsinsuk, P., Analysis and prediction of tyre-soil inter- 
action and performance using finite elements, Journal of Terramechanics, 15(1)(1978), pp. 43-63.

( 5 ) Ueno, M., Hashiguchi, K., Nose, Y., Koyama, F., Uchiyama, K., Izumi, H., Analysis of soil-wheel interaction by elastoplastic finite element method, Proceedings of 10th International Conference of ISTVS, Kobe, (1990), pp. 253-264.

( 6 ) Fervers, C. W., Tyre profile effects on wheel-soil interaction, Proceedings of 12th International Conference of ISTVS, Beijing, (1996), pp. 203-214.

( 7 ) Shoop, S., Finite element modeling of tire-terrain interaction, Technical Report, ERDC/CRREL TR-01-16, (2001).

( 8 ) Oida, S., Seta, E., Heguri, H., Kato, K., Soil/Tire Interaction Analysis Using FEM and FVM, Tire Science and Technology, 33(1)(2005), pp. 38-62.

( 9 ) Cundall, P. A. and Strack, O. D. L., Discrete numerical model for granular assemblies, Géotechnique, 29(1)(1979), pp. 47-65.

(10) Oida, A., Ohkubo, S., Schwanghart, H., Effect of tire lug cross section on tire performance simulated by distinct element method, Proceedings of 13th International Conference of ISTVS, Munich, (1999), pp. 345-352.

(11) Horner, D. A., Peters, J. F., Carrillo, A., Large scale discrete element modeling of vehicle-soil interaction, Journal of Engineering Mechanics, 127(2001), pp. 1027-1032.

(12) Nakashima, H., Fujii, H., Oida, A., Momozu, M., Kawase, Y., Kanamori, H., Aoki, S., Yokoyama, T., Parametric analysis of lugged wheel performance for a lunar microrover by means of DEM, Journal of Terramechanics, 44(2)(2007), pp. 153-162.

(13) Asaf, Z., Shmulevich, I., Rubinstein, D., Predicting soil-rigid wheel performance using distinct element method, Trans. ASABE, 49(3)(2006), pp. 607-616.

(14) Pan, X. D., Reed, M. B., A coupled distinct element-finite element method for large deformation analysis of rock masses, Int. J. Rock Mech. Min. Sci. E Geomech. Abstr., 28(1)(1991), pp. 93-99.

(15) Munjiza, A., The combined finite-discrete element method, John Wiley \& Sons, Chichester, (2004).

(16) Nakashima, H., Oida, A., Algorithm and implementation of soil-tire contact analysis code based on dynamic FE-DE method, Journal of Terramechanics, 41(2/3)(2004), pp. 127-137.

(17) Nakashima, H., Takatsu, Y., Analysis of tire tractive performance on deformable terrain by finite element-discrete element method, Journal of Computational Science and Engineering, 2(4)(2008), pp. 423-434.

(18) Shinone, H., Nakashima, H., Takatsu, Y., Kasetani, T., Matsukawa, H., Improvement of an indoor traction measurement system based on a forced slip mechanism, Proceedings of 16th International Conference of ISTVS, Turin, (2008), pp. 138-143.

(19) Wismer, R. D., Luth, H. J., Off-road traction prediction for wheeled vehicles, Trans. American Society for Agricultural Engineers, 17(1)(1974), pp. 8-10, 14. 